\title{
Destins de la temporalité psychique et survivance après un génocide : une étude de cas à partir de la libre réalisation de l'arbre généalogique
}

\author{
Muriel Katz-Gilbert
}

Dans l'important ouvrage qu'ils consacrent à l'anthropologie du nazisme, Conte et Essner (1995) rappellent la teneur du projet d'extermination des Juifs fomenté par les Nazis. Un projet qui consiste à mettre en œuvre ce qu'ils appellent " la hache généalogique en taillant une frontière de principe entre le "sang juif" et le "sang allemand" " (p. 219). Les auteurs attestent même d'une date et d'un lieu - entre le 9 et le 15 septembre 1935, à Nuremberg, au "Congrès du Parti de la Liberté » - pour situer le temps où la décision fut prise d'user de cette hache généalogique, avec les conséquences irrémédiables que l'on sait puisque celle-ci est à l'origine de l'édiction des lois raciales la même année (Conte et Essner, 1995 ; Hilberg, 1988).

Dans un texte que les auteurs citent, il est question du choix par les nazis d'une charpente sur laquelle se baser pour élaborer le projet nazi. Et cette charpente c'est précisément l'arbre généalogique, seul repère soit disant "fiable " pour évincer le mal désormais qualifié de génétiquement irréversible.

Dans le champ de la psychanalyse, ce sont les analyses de Legendre $(1985,1990,1999)$ sur la question du nazisme qui paraissent faire le plus étroitement écho à l'ouvrage de Conte et Essner (1995). En effet, l'auteur

\footnotetext{
Muriel Katz-Gilbert, maître d'enseignement et de recherche en psychologie clinique, Laboratoire de recherche en psychologie des dynamiques intra- et inter-subjectives (LARPSYDIS), université de Lausanne (Suisse). Chercheuse associée au Centre de recherche français à Jérusalem (CRFJ), psychologue-psychothérapeute.Muriel.Katz@unil.ch
} 
souligne l'importance de la question du généalogique dans la politique anti-sémite qui caractérise l'idéologie du Troisième Reich. Legendre avance la thèse que l'antisémitisme nazi aura produit ce qu'il appelle le "crime généalogique par excellence " (Legendre, 1999, p. 340). "L'extermination consistait, dans son principe, à tuer des fils en tant que fils, les fils de ces fils, [...] les fils de l'un et l'autre sexe, indistinctement donc hommes, femmes, enfants, tous ceux qui font lignée [...] Prétendre éteindre ces lignées, parce qu'elles sont des lignées, qu'est-ce que cela veut dire, en Occident, où ces meurtres ont eu lieu? » demande encore l'auteur (p. 206).

À la suite de ces différents travaux, nous souhaitons explorer comment les sujets qui étaient adolescents, enfants ou encore bébés au moment de la Shoah s'inscrivent fantasmatiquement dans leur lignée généalogique. Comment leurs descendants se pensent-ils eux-mêmes dans la succession des générations qui, avant eux, ont directement subi les persécutions nazies ? Enfin, et plus largement, comment le crime généalogique se répercute-t-il - ou non - dans la représentation fantasmatique qu'un survivant du génocide se fait de sa famille?

Telles sont les différentes questions que nous nous proposons d'explorer dans le cadre d'une recherche interdisciplinaire qualitative ${ }^{1}$ (Katz-Gilbert, 2014, 2015 ; Katz-Gilbert, Bourguignon et Lo Piccolo, 2016 ; Veuillet-Combier et Katz-Gilbert, 2017 ; Katz-Gilbert, Lo Piccolo, Bourguignon et Mariconda, à paraître en 2017).

Certes, nous ne sommes de loin pas les premiers à consacrer des travaux psychanalytiques à la question de la Shoah. Bien d'autres avant nous ont exploré ce champ à partir de ce référentiel théorico-clinique, comme en témoigne l'abondante littérature au sujet de la transmission psychique dans les familles de rescapés, de survivants et de leurs descendants (Barocas $\&$ Barocas, 1973, 1980 ; Bergmann \& Jucovy, 1982 ; Gampel, 2005 ; Kestenberg, 1972, 1980 ; Kogan, 2001 ; Krell, 1979).

L’originalité du dispositif clinique mis en place pour mener à bien ce projet consiste dans le choix d'une médiation projective singulière,

\footnotetext{
1. Les entretiens sont menés auprès de sujets juifs adultes qui pour la plupart sont devenus parents et grands-parents. Les rencontres consistent en un entretien biographique semi-structuré où l'on demande au sujet de raconter son histoire et l'histoire de sa famille, récit complété par un guide d'entretien. Lors d'une deuxième rencontre, le sujet est invité à réaliser librement son arbre généalogique. Nous remercions vivement l'ensemble des personnes qui a d'ores et déjà participé à notre étude dont la poursuite est financée par la Fondation pour la Mémoire de la Shoah, la Fondation Chuard Schmid, la Fondation pour l'Université de Lausanne, la Faculté des Sciences sociales et politiques de l'Université de Lausanne et la Fédération Suisse des Communautés Israélites.
} 
proposée individuellement aux sujets : à savoir la libre réalisation de l'arbre généalogique (Veuillet, 2003a). En écho à la notion de crime généalogique, nous formulons l'hypothèse que cette médiation projective révèle des traces traumatiques relatives à la représentation de la capacité d'engendrer, et partant de transmettre la vie, que ce soit au niveau de l'ascendance ou de la descendance du sujet dont la vie a fait l'objet d'une menace d'extermination génocidaire.

On suppose que les traces en question concernent la fantasmatique originaire du sujet, d'une part, et qu'elles révèleraient d'autre part en creux - inconsciemment donc - certaines impasses identificatoires en lien avec le processus de subjectivation du lien de filiation chez les survivants d'un génocide et leurs descendants (Katz-Gilbert, 2014, 2015 ; Katz-Gilbert, Bourguignon et Lo Piccolo, 2016) ${ }^{2}$.

C'est à travers une étude de cas (Pedinielli, 2011) concernant une femme qui a survécu à la Shoah après avoir été cachée, bébé, avec sa mère que nous nous proposons de montrer l'intérêt d'un tel choix méthodologique. Létude de cas présentée ici sera également l'occasion de souligner l'intérêt d'une référence à la psychanalyse groupale et familiale pour déchiffrer les arbres généalogiques librement réalisés par les sujets.

\section{LA LIBRE RÉALISATION DE L'ARBRE GÉNÉALOGIQUE}

Dans la suite des travaux inaugurés par Lemaire-Arnaud (1980, 1985, 1995) et ses élèves (Savin, 1998), dans la suite de ceux de Santelices (1999) et Veuillet (2003a), nous avons tenté d'explorer la question de la représentation fantasmatique du lien de filiation et, partant, des liens familiaux auprès de sujets qui ont survécu à la Shoah comme de leurs descendants. En effet, le dispositif dit de libre réalisation de l'arbre généalogique (Veuillet, 2003a) n'a, à notre connaissance, pas encore été mis au service de la recherche concernant la violence de masse.

Différent à plusieurs titres du génogramme (Bowen, 1978) tel qu’il est pensé et utilisé par les systémiciens (Katz-Gilbert, Darwiche et Combier, 2015), ce dispositif s'inscrit dans le champ du généalogique et, partant, de la transmission psychique à la fois consciente et inconsciente entre les

2. L'auteure remercie vivement Manon Bourguignon, assistante-doctorante dans notre équipe, pour sa précieuse relecture du manuscrit, ainsi que B. Savin et notre superviseur pour les échanges à propose de cette étude de cas. 
générations. C'est dire si la dimension fantasmatique prime ici sur l'investigation d'une cartographie " objective " de la généalogie.

Si la libre réalisation de l'arbre généalogique constitue à notre sens un dispositif privilégié pour explorer nos questions de recherche clinique, c'est premièrement qu'elle permet d'explorer la manière dont le sujet se représente ses origines. Ce dernier est en effet d'emblée convoqué sur le terrain de la fantasmatique originaire, laquelle est mobilisée par une consigne des plus ouvertes, qui fait écho à la règle de libre association (Katz-Gilbert, 2015 ; Katz-Gilbert, Bourguignon et Lo Piccolo, 2016 ; Lemaire-Arnaud, 1980, 1985, 1995 ; Savin, 1998 ; Savin, Veuillet-Combier et Katz-Gilbert, 2015).

Comprise comme une matrice fantasmatique universelle concernant la question des origines et de la filiation (Racamier, 1992), la scène primitive (Freud, 1915) est abordée dans ce contexte comme une "scène de la transmission par excellence " (Kaës, 2000). Plus largement, ce dispositif donne accès à la représentation fantasmatique des liens familiaux, que ceux-ci renvoient à la filiation ascendante ou descendante, aux liens d'alliance, aux liens fraternels ou encore avunculaires. C'est ce que Eiguer (1987) appelle la "parenté fantasmatique ». Mais c'est également parce que la libre réalisation de l'arbre généalogique permet de mettre en évidence les creux de la transmission (Veuillet, 2003a) - ce que différents auteurs s'accordent à appeler son « versant négatif » (Granjon, 2011 ; Kaës, 2002) - qu'elle nous a semblé particulièrement utile à soutenir notre questionnement.

À la différence du Rorschach ou du TAT, la libre réalisation de l'arbre généalogique ne repose pas sur un matériel pré-établi à la fois " concret " et " ambigu » selon les termes de Chabert (2007, p. 555). Sa spécificité est d'être organisée autour d'un matériel au départ inexistant. Inviter un sujet à réaliser son arbre consiste donc à solliciter la production/création (Katz-Gilbert, 2015) d'un matériel singulier de nature graphique, à propos duquel un échange verbal aura lieu lorsque le sujet estime avoir terminé son tracé.

À travers l'exploration en après-coup des traces graphiques déposées sur le papier, à travers ce que le sujet peut en dire ou non, on tente d'éclairer les processus psychiques en jeu dans la rencontre transféro/contre-transferentielle. En effet, la production/création du sujet est adressée au clinicien mobilisant du même coup une dynamique singulière qui constitue le creuset à partir duquel le matériel recueilli est élaboré (Savin, 1998 ; Veuillet, 2003a ; Katz-Gilbert, 2015).

Nous considérons à la suite de Veuillet (2003a) qu'il s'agit en ce sens d'une médiation projective, structurée en deux phases : graphique tout 
d'abord, puis discursive. Lors de l'échange verbal qui fait suite au tracé, on suppose que les processus secondaires prendront le relais des processus primaires mobilisés, quant à eux, lors de la mise en représentation graphique de la généalogie fantasmatique (Savin, 1998 ; Katz-Gilbert, 2015). Une telle sollicitation permet en ce sens la révélation au sujet lui-même d'une réalité fantasmatique qui surgit au fur et à mesure de son tracé, reflétant la subjectivité de l'auteur (Pedinielli, 2012, p. 61). En ce sens, les notions de déplacement et de condensation caractéristiques du travail du rêve (Freud, 1900) seront utiles au repérage des processus inconscients à l'œuvre dans cette situation projective ${ }^{3}$, comme l'étude de cas qui va suivre permettra de l'illustrer.

Au service du lien entre le clinicien ou le clinicien chercheur et le sujet/ patient, entre le chercheur et son objet de recherche, au service du travail de liaison psychique (Azoulay, 2013a), la libre réalisation de l'arbre généalogique met le processus de symbolisation au travail. C'est en ce sens qu'on peut considérer qu'il s'agit d'un dispositif pour symboliser (Roman, 1997). Le clinicien invite dès lors le sujet à s'engager dans l'« inscription d'une trace graphique à propos de laquelle une discussion est sollicitée » (p. 649), sous la forme d'une " prise de parole très ouverte » en après-coup de son tracé (Chabert et Verdon, 2008, p. 128). Les données recueillies vont alors relever à la fois d'un registre manifeste et d'un registre latent, que ce soit à travers ce que le tracé graphique et le discours donnent à voir et à entendre, ou à travers ce que le sujet omet de représenter et de signifier explicitement ensuite dans l'échange.

Le « visage de l'arbre " (Combier-Veuillet, 2015) prend ainsi progressivement forme, grâce à la mobilisation des processus de symbolisation engagés dans la tâche. Mais les empêchements du travail de symbolisation (Roman, 2007) sont aussi remarquables puisque la production/création graphique comporte également des blancs, signant la présence d'impasses parfois significatives. C'est dire la complexité du matériel recueilli lors de la rencontre qui préside à la libre réalisation de l’arbre généalogique!

Envisagée sous cet angle, cette dernière suppose un cadre qui relève de ce que Azoulay (2013a) propose d'appeler une situation projective, fondée sur une éthique au service de la singularité du sujet et de la protection de sa vulnérabilité psychique. Elle privilégie une « liberté participative originale »

3. À propos du rapprochement entre production onirique et production graphique cf. Savin (1998) ; Veuillet (2003a). 
du sujet (Chabert et Verdon, 2008, p. 128), la prise en compte respectueuse de ses défenses et aménagements psychiques singuliers.

La présente contribution se présente sous la forme d'une étude de cas qui concerne Elsa, une femme qui est aujourd'hui à la fois mère et grand-mère et qui a survécu à la Shoah. Notre entrevue avec Elsa s'inscrit dans le cadre d'une recherche clinique qui compte actuellement plus d'une trentaine de sujets que nous rencontrons individuellement à deux reprises. ${ }^{4}$

L'objectif principal de la présente étude consiste à montrer l'utilité de la libre réalisation de l'arbre généalogique pour explorer les répercussions psychiques de ce que Waintrater (2002) appelle une catastrophe de la filiation: "Tout génocide est une catastrophe de la filiation : il est l'anéantissement simultané de trois générations, celles qui sont nécessaires à l'établissement de toute filiation, pour permettre que chacun puisse se situer dans un ensemble de sujets et se reconnaître comme ayant été engendré et capable d'engendrer » (p. 84).

\section{ÉCHAPPER À LA DÉPORTATION À L’AUBE DE LA VIE}

\section{Observation}

Elsa est née en Belgique en 1944 dans une famille juive originaire d'Europe de l'Est. La mère d'Elsa évite une rafle alors que sa fille était encore en bas âge. Grâce à un réseau de résistants, elles trouvent toutes deux refuge dans un lieu isolé, renfermé et inhabité où elles vivront cachées jusqu'à la Libération. Quant au père d'Elsa, il s'enfuit le premier de son côté non sans avoir organisé la fuite des siens. Elsa et ses parents seront tous trois sauvés et se retrouveront à la fin de la guerre. Ils apprendront alors la déportation des grands-parents maternels d'Elsa en proximité desquels ils habitaient.

Invitée à réaliser son arbre généalogique, Elsa dit d'emblée : «Le seul arbre généalogique que j'ai, c'est du côté de ma mère, du côté de mon père j'ai rien ". Elle poursuit en demandant : "Faut mettre les noms? ". Elle prend la feuille A3 que nous avons placée horizontalement devant elle et la plie en deux. Le côté droit de la feuille recouvre donc entièrement

\footnotetext{
4. La plupart des sujets ont été contactés par courrier par l'intermédiaire de professionnels qui travaillent au sein des communautés, réseaux, institutions et associations juives. Ces derniers ont en effet d'abord pris connaissance des conditions qui sont les nôtres pour participer à cette recherche. À savoir, principalement avoir grandi pendant la Shoah dans un pays qui persécutait les Juifs, être l'enfant d'au moins un parent qui a lui-même fait l'objet de telles persécutions, etc. Ils ont ensuite contacté les personnes concernées pour leur transmettre notre invitation.
} 
le côté gauche qui restera, lui, blanc de part en part. Sur le haut dans la partie droite de la feuille, elle inscrit " grand-père ", "grand-mère ", puis elle note " 5 filles ", c'est-à-dire le nombre d'enfants nés de ses grands-parents maternels.

Figure 1 : arbre généalogique d'Elsa ${ }^{5}$

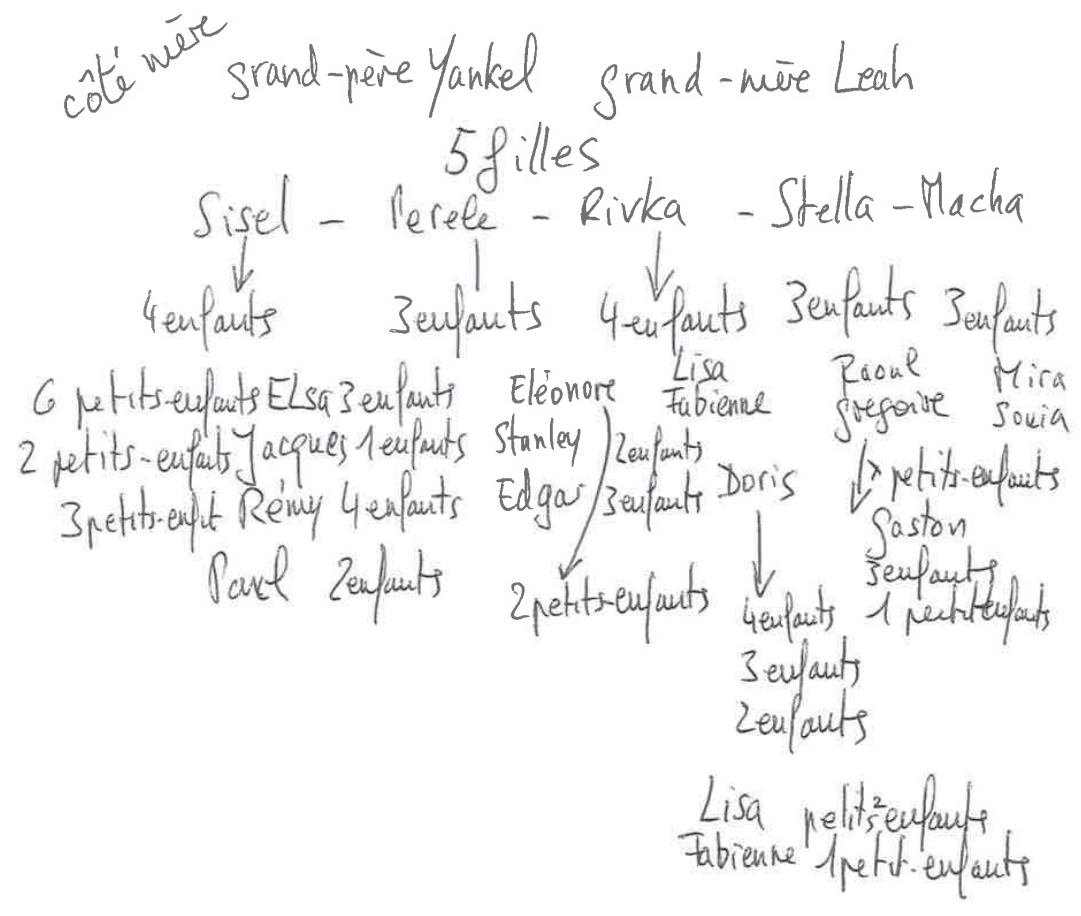

Elle revient ensuite à ses ascendants maternels dont elle inscrit les prénoms (Yankel puis Leah) et poursuit en notant celui de sa mère (Sisel) ainsi que ceux de ses quatre tantes (Perele, Rivka, Stella, Macha). Elle inscrit alors des flèches sous trois de ces prénoms et indique par un chiffre le nombre d'enfants de chacune : 4 enfants, 3 enfants, etc.

Elle note ensuite 3 enfants à droite de son propre prénom et en fait de même pour signifier ses neveux ( 1 enfants (sic), 4 enfants, 2 enfants). Elle procède de manière analogue pour écrire ensuite 6 petits-enfants à gauche de son prénom puis ses petits-neveux. Toutefois, sur cette partie de l'arbre

5. L'arbre ci-joint a été rendu méconnaissable de manière à garantir la confidentialité des données de recherche. Tous les noms ainsi que certains éléments ont été modifiés en ce sens. 
qui représente sa lignée maternelle, elle omet de faire figurer les conjoints, à commencer par son propre mari : l'arbre ne représente donc aucun de ses oncles, ni ses belles-sœurs, ni ses beaux-enfants, etc.

Elle poursuit dans le même sens pour indiquer, toujours par des prénoms, mais aussi par des nombres, des cousins qui sont nés dans sa famille maternelle. Enfin, la dernière information qu'elle fait figurer en haut de l'arbre c'est " côté mère ", juste après avoir dit sobrement " du côté de mon père je ne sais pas".

\section{Commentaire}

Sur un plan contre-transférentiel, la production/création d'Elsa aura suscité la surprise au fur et à mesure de son tracé. Le fait qu'elle commence par plier la feuille en deux pour recouvrir le blanc de sa filiation paternelle en disant « du côté de mon père je ne sais rien » a donné l'impression d'un impensable retour en arrière gelant les affects mobilisés par la tâche et par la rencontre avec la chercheuse clinicienne.

La rêverie sollicitée à notre invitation à propos des origines d'Elsa et plus globalement de ses liens familiaux semble mobiliser d'imposants mouvements défensifs en ce qui concerne la lignée paternelle, mouvements qu'il conviendra d'explorer aussi délicatement que possible en après-coup du tracé.

$\mathrm{Au}$ fur et à mesure qu'Elsa remplit la feuille de noms et de chiffres pour donner forme à sa lignée maternelle, en en faisant scrupuleusement la comptabilité, le visage de l'arbre prend un caractère encombré et confus. Qui est qui ? Comment différencier les nombreuses informations qu'elle tente d'inscrire comme machinalement sur son arbre par ailleurs amputé des conjoints ? Enfin, quel sens donner au sentiment fugitif de profonde tristesse qui nous gagnera à différents moments de la rencontre qui fut souvent émaillée de soupirs et de silence?

\section{À PRopos de LA LIGNÉE PATERNELle... LE CLIVAGE À L'CEUVRE}

Dans la deuxième phase de l'entretien qui consiste à commenter en après-coup du tracé chaque élément graphique, il est d'abord question de ses grands-parents maternels, Yankel et Leah : elle souligne d'emblée qu'il n'était jamais question d'eux en famille. Si elle a connaissance de leurs prénoms et de leur date de naissance, c'est qu'elle a retrouvé des papiers 
officiels dans les documents de famille. Mais ces aïeux n'étaient jamais évoqués. On reconnaît ici la figure du pacte dénégatif (Kaës, 2009) qui scelle parfois la vie des familles touchées par un génocide (Katz-Gilbert, Bourguignon, Lo Piccolo, 2016). La réalité de l'extermination est comme mise sous scellé, empêchant la circulation de la parole et préservant l'unité du groupe famille formé par les survivants et leurs descendants.

Elsa enchaîne ensuite sur le prénom de sa mère : certes, elle a inscrit Sisel sur l'arbre, mais elle précise qu'en fait son prénom d'usage a été officiellement changé après la guerre. Il fallait en effet trouver un prénom qui ferait plus belge : "Il fallait-fallait rentrer dans le moule ", disait à ce propos un des membres de la famille devenu très influent suite à la déportation des grands-parents maternels.

Ce sera donc Louise, toute sa vie durant, même si c'est bien Sisel qui figure sur ses papiers officiels. Elsa précise alors avec un certain cynisme que le souci majeur des siens était la conformité avec les valeurs de la Belgique : ordre, propreté, politesse et assimilation constitueraient le sésame incontournable d'une vie bourgeoise rangée et tranquille.

L'enjeu ? Éviter d'être la cible de l'antisémitisme, explique Elsa, ce qui éclaire aussi l'enjeu du pacte dénégatif en question : rentrer dans le moule du pays d'accueil que représente la Belgique constitue un gage de sécurité pour la survie d'un groupe familial partiellement anéanti durant la Shoah. Cela semble d'autant plus nécessaire que, comme le relate bien Elsa, elle et les siens vivaient en vase clos, dans une communauté juive très soudée, située au cœurs d'une ville où l'antisémitisme de la bourgeoisie catholique était tout à fait sensible.

Elle revient à ses grands-parents, au choix de s'exiler en Belgique entre-deux-guerres depuis leur Hongrie natale pour échapper aux persécutions. "Men ist azoy wie Gott in Belgye! ", dit-elle alors ironiquement en yiddish pour indiquer la désillusion qui fut celle des siens. Une formule à elle, en écho à la formule originale ${ }^{6}$, laquelle souligne pour sa part le rôle exemplaire de la France républicaine dans l'émancipation des Juifs au dixneuvième siècle.

Devenue mère à son tour, elle n'aura pas fait de la conformité au moule une valeur cardinale à transmettre à ses enfants, même si, pour elle et son mari, la continuité compte quand on est Juif. Elle évoque alors pudiquement le fait que son père tenait beaucoup au judaïsme comme culture et

6. «Heureux comme un Juif en France!» 
comme appartenance, d'où l'importance de maintenir les mariages entre Juifs, même si la religion comptait peu pour lui. D'ailleurs elle-même y tient aussi vraiment à cette identité, à cette appartenance qui est la sienne, précise-t-elle.

Avant de rencontrer son époux, elle s'est aussi demandé si elle allait rester en Belgique ou si elle allait aller s'installer en Israël. Elle évoque alors l'antisémitisme des catholiques belges et "l'accent à couper au couteau " des Juifs de sa ville qui contraste avec l'excellent français de son père qui avait appris cette langue enfant. Nous profitons de cette échappée spontanée au sujet de la langue du père pour questionner Elsa sur l'origine sociale de celui-ci qui s'avère élevée. Oui, le fait de maîtriser la langue française aura été un atout qui a joué un rôle significatif dans le choix de s'installer en Belgique. D'ailleurs, précise-elle : "C'est le seul qui est venu en Europe de l'Ouest, les autres ont disparu ", puis rectifie : "Non, non, non les autres sont partis aux États-Unis ». À la question de savoir si ses grands-parents avaient des frères et sœurs, elle répond en indiquant que la famille de sa grand-mère était une famille nombreuse. Ceux qui sont venus en Belgique ont survécu, "les autres on ne sait pas, on ne sait pas...", dit-elle douloureusement.

Ces disparitions de part et d'autre de ses deux lignées ont-elles fait l'objet de recherches après la Shoah, à Yad Vashem par exemple ? Oui, car elle et ses frères auraient voulu documenter les origines du père, mais : " Il n'y a rien, on ne peut rien retrouver de ce côté-là, on ne peut rien, on peut rien retrouver (soupir) il n'y a rien donc euh... même à Yad Vashem non y'a pas... on trouve pas... il n'y a rien, c'est le néant donc comme si cette famille avait jamais existé... Non c'est pas facile, je peux dire ça dans le micro parce que finalement [à mon] âge... on essaie de se représenter ce que ça veut dire de-de pas retrouver...les gens! Il n’y a rien!»

Ces différents éléments discursifs éclairent le sens que l'on peut donner au mouvement défensif qui préside au blanc concernant la lignée paternelle. Ils conduisent de notre point de vue à privilégier une hypothèse concernant une problématique traumatique. Toute une lignée ou presque a en effet été exterminée, apprend-on, sans toutefois qu'on puisse retrouver la trace ni de ces aïeux, ni des conditions de leur mise à mort. Voici de quoi il est principalement question dans l'échange avec la chercheuse clinicienne, dont on rappellera qu'il a lieu dans le contexte d'une recherche où le sujet est invité à revenir sur l'histoire familiale, avec toutes les limites de la situation en question. 
On peut bien entendu se demander dans quelle mesure le clivage pointé ici reflèterait une problématique œedipienne. La remémoration d'éléments associés à la lignée paternelle semble poser problème : le peu d'espace laissé au tiers lors de l'échange témoignerait-il d'un mouvement défensif qui viserait par exemple à se protéger de l'agressivité à l'égard du père et d'une profonde blessure narcissique concernant la figure paternelle?

Envisagé sous cet angle, le versant idéalisant du clivage renverrait quant à lui au rôle présenté comme si capital de la mère d'Elsa dans sa petite enfance. Il n'en reste pas moins que les éléments discursifs dont nous disposons ne permettent pas d'en dire plus sur la problématique œdipienne, qui n’apparaît pas au premier plan dans le discours que livre Elsa en après-coup de son tracé.

Ce que dit Elsa laisse penser que ce mouvement défensif vise avant tout à se protéger de l'impensable disparition des siens évoquée lors de cette rencontre, protégeant par la même occasion Elsa de tout ce qui pourrait par ailleurs susciter la remémoration d'éléments infantiles chargés émotionnellement. Confrontée au vertige que suscite l'évocation d'une histoire familiale fracturée par un crime généalogique, Elsa semble dans l'impossibilité d'aborder ce pan de ses origines qui se révèle comme gelé.

Si l'on suit au contraire le fil associatif d'Elsa, l'espace laissé en blanc figurerait, en négatif, la béance, le néant face auxquels elle se trouve. Elle poursuit d'ailleurs à ce sujet : «Rien... comme si... nous-nous on était nés euh de-de nulle part! Donc euh comme ça euh... comment s'est fait la-la... les générations euh qui ont suivi ? Donc euh... c'est bizarre ! Des propos qui traduisent bien à notre sens l'écho, chez Elsa, de la logique qui sous-tend le délire de filiation caractéristique de l'idéologie nazie. L'impensable néantisation génocidaire fait d'elle une femme qui se présente comme privée d'information et, plus largement, d'une histoire vivante concernant son passé généalogique, ses origines et certains ascendants.

On peut dès lors se demander si l'espace laissé en blanc sur la feuille par Elsa rendrait compte de l'actualité psychique d'une réalité traumatogène brute non élaborée et laissée en héritage, en écho avec « l'angoisse et [le] silence des parents, qui en défaut de représentations communicables [...] transmettent des affects bruts " (Kaës, 2009a). La béance originelle au sujet des ascendants d'Elsa qui ont péri dans la nuit de l'antisémitisme produit des éléments bruts, gelés et énigmatiques, non-symbolisables. C'est ce que Grange-Sénégal (2009) appelle les « choses de la transmission». 
La partie laissée en blanc sur la feuille peut dès lors être comprise comme l'irreprésentable présence d'une absence, révélée grâce à la dimension projective de l'outil. Innombrables, les victimes familiales du nazisme ressemblent dès lors à des fantômes hantant la rencontre, asséchant du même coup la vie des affects de par leur caractère irreprésentable ${ }^{7}$.

On considérera en ce sens cette omission, ce blanc concernant la lignée paternelle comme une trace « laissée en négatif par l'objet» (Roman, 1995). Un blanc qui serait par ailleurs contre balancé par l'inscription répétitive et foisonnante des descendants et dont on interrogera par la suite le caractère compulsif.

\section{À PROPOS DES LIENS D'ALLIANCE... LE DÉPLACEMENT À L'CEUVRE}

Mais outre le blanc qu'il engendre, l'ampleur du clivage est tel qu'il désorganise la structure générale de l'arbre. Aucun couple n'est en effet figuré sur l'arbre, exception faite des grands-parents maternels, dont elle rapportera quelques éléments en ce qui concerne leur fin tragique. On reconnaît ici, avec Altounian (1990), la mise en évidence d'une génération d'ancêtres - celle qui a vécu le génocide - et qui, sur le plan fantasmatique, est présentée comme inaugurant une nouvelle lignée.

Certes, Elsa mentionne oralement l'existence de son père et des siens, ce qui permet d'exclure une problématique relevant de la forclusion associée à la psychose. Mais à partir de la fratrie d'Elsa, tous les enfants figurés sur l'arbre naissent fantasmatiquement d'un seul parent, souvent d'une femme, donc la mère, mais pas uniquement.

Point de père, point d'oncles maternels, point de mari pour Elsa, point de beaux-frères, ni de belles-sœurs sur cette production/création! Bref, pas de conjoints, alors que l'arbre comptabilise par ailleurs les nombreux petits-enfants, neveux et nièces et petits-neveux et nièces d'Elsa, mais sans toutefois qu'on puisse repérer de quelconques couples parentaux. Lors de la discussion, celle-ci n'évoquera d'ailleurs presque jamais spontanément les conjoints.

Sur le plan latent, l'éviction quasi systématique des conjoints ainsi que des indices graphiques permettant de figurer les liens d'alliance interroge en profondeur. On peut se demander s'il ne s'agit pas de l'effet d'un important déplacement du blanc concernant la lignée paternelle et partant, de la scène primitive, du côté gauche vers le côté droit de la feuille.

7. D’où la fécondité de la notion de métapsychologie de l'absence (Golse, 1995). 
On est ainsi amené à faire l'hypothèse qu'un fantasme inconscient de parthénogenèse traverse cet arbre en filigrane (Guyotat, 1980, 1995). Par parthénogenèse, on entend une reproduction sans fécondation, autrement dit une reproduction monoparentale à partir de la mère ${ }^{8}$ (Guyotat, 1995, 2005). "Un vient de un ", dans cette perspective (Ansermet, 1999, p. 32). On reconnaît les traits d'un système de reproduction unisexuée, caractéristique d'une représentation narcissique du lien de filiation.

Or, un tel fantasme gagne sans doute ici à être éclairé sous deux angles : d'une part, et même si cela n'a pas toujours été le cas, le judaïsme est conçu comme se transmettant par la mère. On voit ainsi se dessiner le rôle central de la filiation maternelle dans cette tradition. Mais le déficit du lien institué, caractéristique des événements symbolicides qui sous-tendent la filiation traumatique, permet également d'éclairer le fantasme en question (Guyotat, 1995, 2005). À partir de la génération mise au monde par le couple des grands-parents maternels assassinés par les Nazis, à partir de la Shoah donc, on semble enfanter seul, de génération en génération, dans la famille fantasmatique représentée sur le papier.

Telle qu'elle est figurée dans cet arbre, la transmission de la vie semble donc passer outre la différence des sexes et des générations qui structure au contraire, par définition, la reproduction sexuée. Sur le plan fantasmatique, la logique narcissique-imaginaire prend le pas sur la logique-symbolique qui structure la filiation instituée. Car si la filiation instituée est médiatisée par la Loi, par les grands interdits fondateurs (Guyotat, 1980 ; 1995), par ce que Kaës (2009a) appelle les "garants métapsychiques » au niveau collectif, c'est au contraire leur effondrement qui ouvre la brèche à la logique narcissique-imaginaire.

L'inflation narcissique dont témoigne la production/création d'Elsa peut en ce sens être comprise comme l'effet d'une régression associée à la situation projective. Les flèches tracées à partir de la fratrie de Sisel, la mère d'Elsa, traduisent peut-être à la fois la place centrale de la mère dans la transmission de la judéité et le rôle vital de la mère d'Elsa dans les premiers mois de sa vie. Une place, un rôle qui se présentent ici comme survalorisés au détriment de la place réservée au tiers.

8. Ce terme, courant en biologie, vient du grec parthenos qui signifie «vierge». Le mythe de l'«Immaculée conception » figure de manière emblématique ce qu'on entend par parthénogenèse. 
Ces commentaires conduisent à formuler l'hypothèse que les aménagements défensifs déployés dans la rencontre peuvent être compris en lien avec la difficulté d'Elsa à revenir sur ce passé inaugural douloureux et probablement traumatique pour sa mère, à en croire entre autres le silence dont il a été entouré.

La conclusion qu'elle tiendra spontanément à donner à l'entretien permet d'ailleurs d'aller dans ce sens. Elle évoque en effet la dette incommensurable qu'elle ressent par rapport à sa mère, aujourd'hui très âgée et invalide : "En fait, euh, elle, elle avait tout fait pour me maintenir en vie, elle, elle a risqué sa vie... pour moi ", dira-t-elle sobrement et non sans émotion. On entrevoit ici l'actualité d'un fantasme d'être l'enfant parthénogénétique de la mère, ce qui ne manque pas de questionner.

Exprimé dans le contexte où Elsa est invitée à évoquer son histoire précoce, un tel fantasme peut-il être compris sous l'angle de sa fonction défensive "vitale ", au service de ce que Racamier appelle le "principe de survivance " par contraste avec le " principe d'anéantissement " ? Évoquant la menace externe et traumatogène qu'aura représenté le fait d'être traquées/ cachées, Elsa est-elle aux prises avec le fait que survivre à la menace de l'autre renvoie aussi à la menace interne d'auto-anéantissement (Kaës, $2009 b$ ) ? Serait-on en ce sens en présence d'une forme de fixation à un noyau traumatique qui permettrait d'éclairer l'actualité d'un fantasme obéissant à une logique narcissique?

La suite du discours permet en tous les cas de souligner le poids de la dette qu'Elsa éprouve envers sa mère : aujourd'hui encore, précise-t-elle, elle se sent profondément redevable à celle à qui elle dit devoir sa survie, mais sans toutefois être à même de témoigner en retour à sa mère les soins qu'elle aurait voulu pouvoir lui donner au soir de sa vie. Nous proposons d'appeler cette dette incommensurable dont il est ici question, "dette de survie ", avec tous les mouvements d'idéalisation et de réparation qu'elle recouvre. Une dette qui, au même titre que la dette de vie, dont la subjectivation est essentielle au devenir parent (Bydlowsky, 2008), appellerait une élaboration pour pouvoir être dépassée.

L'enjeu est de taille puisque la question de la réparation semble ici au cœur de celle de la transmission de la vie, ce dont la production d'Elsa ainsi que son discours semblent témoigner en lien avec le contrat narcissique originaire dont il sera question ci-dessous. 


\section{LA COMPULSION EN QUESTION}

Après l'évocation des recherches infructueuses concernant les origines du père, nous tentons d'y voir plus clair concernant la génération de ses grands-parents, suivant ainsi le fil associatif du discours d'Elsa. Elle évoque alors une anecdote concernant, dit-elle, son grand-père maternel : il avait l'habitude de passer la main sur la tête de chacun de ses nombreux enfants le soir, ce que la mère d'Elsa prenait pour une bénédiction alors qu'il s'agissait simplement de les compter pour vérifier qu'ils étaient " tous bien là ». À ce moment-là, les générations se mélangent entraînant un important flou ; la confusion nous gagne sur le plan contre-transférentiel, sentiment que nous reflétons à Elsa sous la forme d'un questionnement sur l'ordonnancement des générations. Elle réalise alors la confusion générationnelle inhérente à son propos et rectifie : c'est sa grand-mère Leah qui racontait cette histoire à propos de son propre père, l'arrière grand-père d'Elsa.

Dans l'après-coup de son tracé, cette anecdote et la confusion qui l'aura entourée nous apparaît comme significative à plusieurs égards. En effet, nous avons été frappée par l'important contraste entre le blanc général de l'arbre concernant la lignée paternelle d'une part, et le foisonnement des descendants comptabilisés en détail du côté maternel, d'autre part. On peut dès lors s'interroger sur le caractère compulsif de la comptabilité à laquelle Elsa se livre non seulement concernant ses propres enfants, mais également eu égard à l'ensemble des descendants issus de la lignée maternelle. Identifiée à son arrière-grand-père maternel, Elsa se livrerait-elle ainsi à une comptabilité qui permet de vérifier que " tout le monde est bien là " dans un mouvement fantasmatique défensif de compensation des pertes innombrables que représente la Shoah pour sa famille?

L'hémorragie de descendants contraste en effet clairement avec l'omission des nombreux disparus du côté paternel. Ici les descendants semblent devoir affluer en masse sur le plan fantasmatique comme pour contre-balancer l'impossible évocation des innombrables disparus laissés sans sépulture (Gilbert, 2005). Un blanc qui fait aussi penser à un « linceul de silence " selon la précieuse expression de Drieu, Zanello, Proia-Lelouey (2009, p. 122).

On est frappé par la place que prennent ces nombreux descendants dans l'économie générale de l'arbre d'Elsa et les fort nombreuses précisions quantitatives concernant la filiation descendante, d'une part et par le caractère horizontal de l'inscription de celle-ci, d'autre part. À la suite de 
Tuil (2005), on peut interpréter la disposition en ligne comme un nécessaire corps-à-corps. Faire corps pour faire groupe, écrit-elle, "dans un ordre qui privilégie la contiguiité au détriment de la continuité et de la succession des générations" (p. 131).

Le dénombrement prend clairement le pas sur la différenciation des places et des sexes de chacun : si la fratrie d'Elsa est prénommée après avoir été comptabilisée, les enfants d'Elsa, comme ses petits-enfants ne reçoivent en revanche pas de prénom sur son arbre. On ne connait du coup ni leur sexe, ni leur ordre de naissance, ni leur âge. L'indication de leur nombre borde en outre le prénom d'Elsa, leur mère et grand-mère, comme s'ils lui donnaient des ailes, des bras, comme s'ils la complétaient de part et d'autre. Une singularité graphique qui interroge : ce rapproché, pour ne pas dire ce collage, renverrait-il à des objets partiels vécus comme une part de soi ? Peut-on l'interpréter comme la trace d'une survie miraculeuse, celle d'Elsa, qui serait destinée à se reproduire à l'envi sous la forme d'un clonage fantasmatique?

On note aussi que ces indications chiffrées prennent la place habituelle du conjoint, comme souvent sur l'ensemble de l'arbre. Les temps se confondent, les chiffres remplacent les prénoms et les noms, brouillant du même coup leur différenciation sexuée et générationnelle. La chronologie des naissances est ainsi aplatie, les enfants et les petits-enfants d'Elsa se retrouvant au même "étage " généalogique qu'elle, c'est-à-dire à ses côtés, dans une contiguïté qui interroge.

Le dénombrement a donc pris le pas sur l'inscription historicisante de chacun, évinçant du même coup la dynamique propre à l'écoulement linéaire du temps de l'existence humaine. On reconnaît ici, avec Azoulay (2013b), les traits singuliers d'une souffrance associée à l'impensable perte, qui renvoie à notre sens également à l'absence d'indices permettant de différencier les morts des vivants dans l'arbre d'Elsa.

En effet, rien ne permet de repérer, sur le papier, que les grands-parents maternels d'Elsa figurés en haut de la production/création ont été assassinés. Ils sont là, régnant sur leur descendance, comme s'ils étaient encore bien vivants, ce qui illustre bien le caractère zeitlos des processus primaires mobilisés au moment de réaliser librement un arbre généalogique. Une présence tutélaire qui en dit long sur la nécessité de compenser l'absence de tant d'aïeux, à commencer par les grands-parents paternels, sans doute absorbés dans le blanc général qui caractérise le côté gauche de la feuille. 
Envisagée sous cet angle, la prolifération graphique de descendants peut être considérée comme l'expression d'une défense mobilisée pour faire face aux pertes innombrables dues à la Shoah et aux blancs qu'ils entrainent, compromettant du même coup le travail de symbolisation et de différenciation. Une comptabilité graphique qui fait penser à un mouvement de compensation psychique.

\section{LE CONTRAT NARCISSIQUE ORIGINAIRE EN QUESTION}

Au niveau groupal, le visage singulier que prend cet arbre (Combier, 2015) permet d'avancer une dernière interprétation : pour Elsa et sa génération, engendrer aura constitué une mission vitale qui est projetée sur le papier, non seulement en ce qui la concerne, mais en ce qui concerne l'ensemble de son groupe-famille à partir de la génération de sa mère et de ses tantes maternelles.

La notion de " contrat narcissique originaire " (Kaës, 2009a) paraît ici utile pour éclairer un des possibles sens de la mission compensatrice en question. Il s'agit en effet d'un contrat qui, dans la suite de Racamier, vise à sceller "l'affiliation narcissique " des sujets à l'" espèce » humaine (p. 61). Or, c'est précisément un tel contrat qui fait l'objet de la barbarie génocidaire, laquelle tend à déshumaniser un peuple tout entier en l'anéantissant (Kaës, 2009). On peut donc se demander dans quelle mesure Elsa ne saisit pas l'occasion de cette rencontre pour tenter de restaurer l'image groupale inconsciente, autrement dit l'imago familiale.

Le fait que l'arbre ne comporte aucun patronyme permet de soutenir une telle hypothèse. En effet, l'indication du nom de famille renvoie à la filiation instituée, autrement dit à la logique symbolique. L'absence de tels indices peut être interprétée comme significative d'un événement symbolicide, dans la mesure où le crime de masse suppose l'effondrement de ce que Zaltzman (2007) appelle « le pacte d'identification à l'espèce humaine » et à ce que Kaës $(2009,2012)$ appelle « l'effondrement des garants métapsychiques » sur lesquels repose précisément la tiercéité associée à la Loi (Hirsch, 2015).

S'agirait-il ainsi d'un mouvement défensif de réparation qui fait écho à ce que Waintrater (2002) appelle une " catastrophe de la filiation " ? Dans quelle mesure Elsa investit-elle la production/création graphique sollicitée par la clinicienne en endossant le rôle de porte-voix (Kaës, 2012), de portedeuil (Petrou, 2016) ? Par son tracé, par ses paroles, elle s'exprimerait non 
seulement au nom de son groupe-famille, mais aussi au nom du peuple dont elle fait partie et qu'on a tenté d'exterminer. On voit ici combien ce dispositif clinique s'avère précieux pour éclairer la nature des alliances inconscientes, ce qui permet d'explorer l'articulation significative entre les espaces intra-, inter- et transsubjectifs (Kaës, 2009a).

On devine ainsi, en sotto voce, le désir irrépressible de restauration narcissique d'une survivante à l'égard de son groupe-famille et du peuple auquel elle appartient. Un bébé qui, contrairement à tant d'autres enfants morts parce que " fils et filles de ", aura survécu à l'impensable crime contre l'humanité que constitue la Shoah, tout en portant en elle l'héritage d'une présence immémoriale : celles des innombrables morts laissés sans sépulture parmi les siens (Gilbert, 2005).

Arrivé au terme de l'exploration de cette étude de cas, nous avons ainsi été conduite à formuler une seconde ligne d'hypothèses : on suppose en effet que plus les repères identificatoires concernant la différenciation des morts et des vivants sont mis à mal, plus la représentation subjective de la capacité d'engendrer et de transmettre la vie présentera des singularités.

\section{POUR CONCLURE}

Partant d'une hypothèse générale concernant le possible repérage de la filiation traumatique à travers la libre réalisation de l'arbre généalogique, nous avons exploré une production/création sous la forme d'une étude de cas.

Cette dernière conduit à interpréter l'arbre d'une femme qui a survécu, bébé, à la Shoah comme une illustration singulière des deux destins possibles de la temporalité traumatique : le clivage associé au gel des affects en écho à un effet de déliaison psychique d'une part, et la répétition compulsive d'un plaisir associé à une inquiétante énigme, d'autre part.

Ce que le visage de l'arbre singulier d'Elsa permet ainsi de repérer, en termes de fantasmatique originaire, c'est une importante mise à mal de la dynamique œedipienne. Une première hypothèse aura permis d'éclairer le matériel recueilli ainsi que les mouvements transféro-contre-transférentiels en jeu sous l'angle du traumatisme. Le fantasme de parthénogenèse qui traverse l'arbre d'Elsa peut dès lors être compris comme l'expression de ce que Drieu $(2001 ; 2004)$ conçoit comme un « complexe traumatique de filiation ». Cet auteur suppose en effet que l'effroi associé aux traumatismes non élaborés par les générations antérieures se répercuterait - en creux - chez les descendants, imprégnant du même coup leur caractère 
mortifere aux liens fantasmatiques. La recherche de l'unisson narcissique (Racamier, 1995) prendrait dès lors le pas sur l'ouverture à une structuration œdipienne (Drieu et Marty, 2005).

On voit ainsi l'utilité de la libre réalisation de l'arbre généalogique pour explorer les singularités de la filiation traumatique suite à un génocide. Une médiation projective qui permet de plonger de manière originale au plus près de l'hétérochronie fondamentale (Green, 2000). Explorant ce que l'on pourrait considérer comme un témoignage à la fois graphique et discursif, le sillon creusé à l'interne par la pulsion de mort serait dès lors retraçable, ce qui permet d'éclairer la question des possibles singularités associées à la fantasmatique originaire chez des survivants d'un meurtre de masse et leurs descendants. Reste que l'investigation de ces questions appellerait bien entendu d'autres outils projectifs en complément pour explorer leur complexité de manière approfondie.

\section{BIBLIOGRAPHIE}

Abraham, N. \& Torok, M. (1978). L'écorce et le noyau. Paris : Aubier-Flammarion. Altounian, J. (1990). Ouvrez-moi seulement les chemins d'Arménie... Un génocide aux déserts de l'inconscient. Paris : Les Belles-Lettres.

Ansermet, F. (1999). Clinique de l'origine. L'enfant entre la médecine et la psychanalyse. Paris/Lausanne : Payot.

Azoulay, C. (2013a). Les épreuves projectives au service de la liaison, Le Carnet PSY 3, (170), 22-24.

Azoulay, C. (2013b). Temporalité psychique et psychologie projective, Le Carnet PSY, 2 (169), 34-37.

Barocas, H. A. \& Barocas, C. B. (1973). Manifestations of Concentration Camp Effects on the Second Generation, American Journal of Psychiatry, 130, 820-821.

Barocas, H. A. \& Barocas, C. B. (1980). Separation-Individuation. Conflits in Children of Holocaust Survivors, Journal of Contemporary Psychotherapy, 11, 6-14.

Bergman, M. S. \& Jucovy, M. E. (1982). Generations of the Holocaust. New York : Columbia University Press.

Bowen, M. (publié sous : Anonymous). (1978). On the Differentiation of Self. Dans J. Aronson, Family Therapy in Clinical Practice, USA, 1978. [Trad. française, 1993, À propos de la différenciation de soi à l'intérieur de sa propre famille, Thérapie Familiale, 15(2), 99-148.] 
Bydlowsky, M. (2008). La dette de vie. Itinéraire psychanalytique de la maternité. Paris : Presses Universitaires de France.

Chabert, C. (2007). Les épreuves projectives en psychopathologie. Dans R. Roussillon et coll. (dir.), Manuel de psychologie et de psychopathologie clinique générale (p. 555-581). Paris: Masson.

Chabert, C. \& Verdon, B. (2008). Psychologie clinique et psychopathologie. Paris : Presses Universitaires de France.

Combier, C. (2015). Libre réalisation de l'arbre généalogique et clinique de la honte. Dans M. Katz-Gilbert, J. Darwiche, C. Veuillet-Combier (dir.), Génogramme ou arbre généalogique. Approche systémique et psychanalytique (p. 119-139). Paris : In Press.

Conte, E. \& Essner, C. (1995). La quête de la race. Une anthropologie du nazisme. Paris : Hachette.

Drieu, D. (2001). La traumatophilie à l'adolescence - Enjeux thérapeutiques. Thèse de doctorat soutenue à l'Université Denis Diderot. Paris VII.

Drieu, D. (2004). Automutilations, traumatophilie et enjeux transgénérationnels à l'adolescence, Adolescence, 48, 311-323

Drieu, D. \& Marty, F. (2005). Figures de la filiation traumatique, Dialogue, 2 (168), 5-14.

Drieu, D., Zanello, F., \& Proia-Lelouey, N. (2009). Secrets de famille, auto engendrement négatif et enjeux thérapeutiques, Cahiers de psychologie clinique, 1 (32), 119-138.

Eiguer, A. (1987). La parenté fantasmatique. Paris : Dunod.

Freud, S. (1900). L'interprétation des rêves. OC, 2004 volume IV, 1899-1900.

Freud, S. (1915). Leçons d'introduction à la psychanalyse. Paris : Presses Universitaires de France, 2013.

Freud, S. (1926). Inhibition, symptôme, angoisse. Paris : Presses Universitaires de France, 2009.

Freud, A. (1936). Le Moi et les mécanismes de défense. Paris : Presses Universitaires de France, 2009.

Gampel, Y. (2005). Ces parents qui vivent à travers moi: les enfants des guerres. Paris : Fayard.

Gilbert, M. (2005). (sous la direction de). Antigone et le devoir de sépulture. Genève : Labor et Fides.

Golse, B. (1995). "Le concept de transgénérationnel », Carnets psy, 6, 12-16.

Grange-Ségéral, E. (2009). Les « choses » de la transmission. Dans M. Sassolas (sous la direction de). Transmissions et soins psychiques (p. 187-200). Toulouse : érès.

Granjon, E. (2011). La thérapie familiale, lieu d'élaboration du transgénérationnel. Dans J.-B. Chapelier, J. Roffat (dir.), Groupe, contenance et créativité (p. 191-202). Toulouse : érès. 
Green, A. (2000). Le temps éclaté. Paris : Minuit.

Guyotat, J. (1980). Mort/naissance et filiation. Études de psychopathologie sur le lien de filiation. Paris : Masson.

Guyotat, J. (1995). Filiation et puerpuéralité, logique du lien. Entre psychanalyse et biomédecine. Paris : Presses Universitaires de France.

Guyotat, J. (2005). Traumatisme et lien de filiation. Dialogue, 168, 15-24.

Hilberg, R. (1988). La destruction des Juifs d'Europe. Paris : Fayard (édition originale publiée en 1985).

Hirsch, D. (2015). Travail du négatif dans les traumas collectifs et malaise actuel dans la culture. Dans R. Kaës (dir.), Crises et traumas à l'épreuve du temps. Le travail psychique dans les groupes, les couples et les institutions (p. 41-68). Paris : Dunod.

Kaës, R. (2000). Filiation et affiliation. Quelques aspects de la réélaboration du roman familial dans les familles adoptives, les groupes et les institutions. Le Divan familial, 5, 61-78.

Kaës, R. (2002). Le problème psychanalytique du générationnel. Objets, processus et dispositifs d'analyse. Filigrane, 11 (1), 109-120.

Kaës, R. (2009a). Les alliances psychiques inconsciente. Paris : Dunod.

Kaës, R. (2009b). Le travail de l'intersubjectivité et la polyphonie du récit dans l'élaboration de l'expérience traumatique. Dans A. et J. Altounian (dir.), Mémoire du génocide arménien : héritage traumatique et travail analytique (p. 209-235). Paris : Puf.

Kaës, R. (2012). Le Malêtre. Paris : Dunod.

Katz-Gilbert, M. (2014). Du crime généalogique à l'impossible maillage généalogique : à propos de quelques effets de l'antisémitisme nazi sur la subjectivation aujourd'hui. Dans M. G. Wolkowicz (dir.), Présence de la Shoah et d'Israël dans la pensée contemporaine (p. 369-394). Paris : In Press.

Katz-Gilbert, M. (2015). Différencier les logiques de filiation à l'œuvre dans la libre réalisation de l'arbre généalogique. Esquisse psychanalytique à partir d'une recherche sur la Shoah. Dans M. Katz-Gilbert, J. Darwiche, C. Veuillet-Combier (dir.), Génogramme ou arbre généalogique. Approche systémique et psychanalytique (p. 197-238). Paris : In Press.

Katz-Gilbert, M., Darwiche, J., \& Veuillet-Combier, C. (dir.). (2015). Génogramme ou arbre généalogique. Approche systémique et psychanalytique, Paris : In Press.

Katz-Gilbert, M., Bourguignon, M., \& Lo Piccolo, G. (2016). Filiation catastrophique et travail de mémoire après la Shoah : quand la libre réalisation de l'arbre généalogique est au service de l'historicisation. Dialogue, 3 (123), 69-82.

Katz-Gilbert, M. (2017a). Tisser des liens d'affiliation au groupe comme rempart au meurtre de masse : la circoncision en question. Dans C. Combier Veuillet, 
\& E. Gratton (dir.), Figures de la filiation (p. 129-142). Rennes : Presses Universitaires de Rennes.

Katz-Gilbert, M. (2017b). Circumcision ? yes but... ! The study of 'bricolage' in modern Judaïsm from a psycho-analytical perspective. Dans S. Nizard, M. Gross, Y. Scioldo-Zürcher (dir.), Gender, Families and Transmission in Contemporary Jewish Context (p. 158-169). Cambrigdge (GB) : Cambridge Scholars Publishing.

Katz-Gilbert, M., Lo Piccolo, G., Bourguignon, M., \& Mariconda, G. (2017c). Le génocide pensé comme passage à l'acte de nature fanatique : quelles répercussions psycho-sociales ? Une étude de cas menée à partir de la libre réalisation de l'arbre généalogique auprès d'un survivant de la Shoah (à paraître en 2017 dans Cahiers de psychologie clinique).

Kestenberg, J. (1972). Psychoanalytic contributions to the problem of children of survivors from nazi persecutions. The Israel Annals of Psychiatry and Related Disciplines, 10, 311-325.

Kestenberg, J. (1980). Psychoanalyses of children of survivors from the Holocaust: case presentation and assessment. Journal of the American Psychoanalytic Association, 28, 775-804.

Kogan, I. (2001). Le cri des enfants sans voix. L'Holocauste et la deuxième génération: une perspective psychanalytique; Lonay: Champs psychanalytiques.

Kohut, H. (1971). The analysis of the Self. New York : International Univesitary Press.

Krell, R. (1979). Holocaust Families: The Survivors and their Children. Comprehensive Psychiatry, 20 (6), 560-568.

Legendre, P. (1985). L'inestimable objet de la transmission. Étude sur le principe généalogique en Occident. Leçons IV. Paris : Fayard.

Legendre, P. (1990). L'attaque nazie contre le principe de filiation. Dans A. Papageorgiu-Legendre (dir.), Filiations fondement généalogique de la psychanalyse. Leçons IV, suite 2 (p. 205-209). Paris : Fayard.

Legendre, P. (1999). La Brèche. Remarques sur la dimension institutionnelle de la Shoah. Dans P. Legendre (dir.), Sur la question dogmatique en Occident: aspects théoriques (p. 339-349). Paris : Fayard.

Lemaire-Arnaud, E. (1980). "A propos d'une technique nouvelle : le génogramme ", Dialogue, 138, 29-37.

Lemaire-Arnaud, E. (1985). Utilité du génogramme pour la mise à jour des phénomènes transgénérationnels, Dialogue, 89, 3-7.

Lemaire-Arnaud, E. (1995). Le génogramme en thérapie de couple et en thérapie familiale, Dialogue, 130, 47-52.

Pedinielli, J.-L. (2011). L'observation clinique et l'étude de cas. Paris : Armand Colin Pedinielli, J.-L. (2012). Introduction à la psychologie clinique. Paris : Armand Colin. 
Petrou, M. A. (2016). Une déchirure dans la mémoire qui s'agrandit avec le temps. Revue française de psychanalyse, 80 (2), 474-487.

Racamier, P.-L. (1992). Le génie des origines. Psychanalyse et psychose. Paris : Payot. Racamier, P.-L. (1995). L'inceste et l'incestuel. Paris : Éditions du Collège.

Roman, P. (1995). "Du blanc des origines à l'origine du blanc dans la création picturale moderne et contemporaine ", Cahiers de psychologie clinique, 4, 79-90.

Roman P. (1997). « La méthode projective comme dispositif à symboliser : enjeux cliniques et psychopathologiques ». Dans P. Roman (dir.), Projection et symbolisation chez l'enfant (p. 37-51). Lyon : Presses Universitaires de Lyon.

Roman, P. (2007). Figures du négatif et «expérience " créatrice. Dans A. Brun, J.M. Talpin (dir.) Clinique de la création (p. 117-140). Bruxelles :De Boeck.

Santelices, M.-P. (1999). Le génogramme libre et la transmission générationnelle: travail réalisé auprès d'enfants placés. Thèse de doctorat soutenue à l'Université Lumière-Lyon-2, Lyon, France.

Savin, B. (1998). L'écoute généalogique. Son importance diagnostique et thérapeutique en clinique individuelle familiale et institutionnelle (Tome I et II). Thèse de doctorat soutenue à l'Université Lumière-Lyon-2, Lyon, France: Atelier national de reproduction des thèses.

Savin, B., Combier-Veuillet, C., \& Katz-Gilbert, M. (2015). Retrouver ses racines derrière les barreaux : l'investigation généalogique chez un patient toxicomane. Dans M. Katz-Gilbert, J. Darwiche, C. Veuillet-Combier (dir.), Génogramme ou arbre généalogique. Approche systémique et psychanalytique (p. 141-174). Paris : In Press.

Tuil, S. (2005). De l'emploi du génogramme libre en entretiens familiaux à visée thérapeutique. Dialogue, 2 (168), 115-133.

Veuillet, C. (2003a). Adoption et violence de la transmission psychique. Clinique des couples adoptants et enjeux inconscients inter et transgénérationnels. Thèse de doctorat soutenue à l'Université Lumière-Lyon-2, Lyon, France.

Veuillet, C. (2003b). Entretiens psychologiques préalables à l'adoption et « libre réalisation de l'arbre généalogique ». Psychologie clinique et projective, 1 (9), 353-367.

Veuillet-Combier, C., \&Katz-Gilbert, M. (2017). De la mémoire trouée à la parentalité empêchée : clinique de la transmission psychique après un génocide. Revue de psychothérapie psychanalytique de groupe, 68, 195-207.

Waintrater, R. (2002). À la recherche d'une nouvelle filiation. La problématique narcissique dans les groupes de formation au recueil de témoignage de la Shoah. Revue de psychothérapie psychanalytique de groupe, 1 (38), 37-53.

Waintrater, R. (2009). Le temps de l'extrême : génocide et temporalité. Revue de d'histoire de la Shoah, 190, 407-427.

Zaltzman, N. (2007). L'esprit du mal. Paris : L’Olivier. 